\title{
White paper on imaging biomarkers
}

\author{
European Society of Radiology (ESR)
}

Received: 11 May 2010 / Accepted: 11 May 2010/Published online: 27 May 2010

(C) European Society of Radiology 2010

\begin{abstract}
Because of the importance of biomarkers within medicine as a whole and the increasing realisation that imaging can safely provide biomarkers, the ESR executive commissioned this report by experts in the field. It is hoped that further awareness amongst our community will contibute to further exploitation of the enormous wealth of biomaker information available in our day to day imaging. The all important issues surrounding validation and standardisation are discussed, together with proposals for a European Network on Imaging Biomarkers to oversee such aspects.
\end{abstract}

Keywords Imaging · Biomarkers · Pharmacodynamics .

Standardisation $\cdot$ Validation

\section{Introduction}

Biomarkers are characteristics that are objectively measured as indicators of normal biological processes, pathological changes, or pharmaceutical responses to a therapeutic intervention [1]. Depending on the mode of collecting the information, three types of biomarkers can be distinguished:

1. biochemical or histological parameters detected on tissue samples obtained at biopsy or surgery,

2. biochemical parameters or cells obtained on blood or urine samples,

3. anatomical, functional or molecular parameters detected with imaging.

European Society of Radiology (ESR) ( $₫)$

Neutorgasse 9/2,

AT-1010 Vienna, Austria

e-mail: communications@myESR.org
Biomarkers have multiple applications, including:

- Prediction: use as a tool to predict patients at risk of disease. Calcium scoring as an indicator of the risk of coronary artery disease is an example of predictive biomarker.

- Detection: use as a diagnostic tool for the identification of patients with disease. For example, measurement of the hepatic perfusion index at CT has been proposed as a biomarker for the detection of occult liver metastases from colorectal cancer.

- Staging: use as a tool for classification of the extent of disease. PET-CT with measurements of specific uptake values (SUV) is increasingly used as staging biomarker of various malignant tumors.

- Grading: use as an indicator of disease aggressiveness and prognosis. Several studies with variable results have assessed the correlation between MRI perfusion and diffusion parameters and tumor aggressiveness or prognosis.

- Assessment of response to treatment: use as a tool for evaluating the disease response to treatment.

Imaging biomarkers are useful for all these applications. Compared with biochemical and histological biomarkers, imaging biomarkers have the advantage of remaining noninvasive and being spatially and temporally resolved. Imaging biomarkers play an increasing role in major medical areas such as cancer, cardiovascular diseases, neurological and psychiatric diseases, musculoskeletal diseases, metabolic diseases, and inflammatory and autoimmunity-based diseases [2].

\section{Drug development and imaging biomarkers}

One area where imaging biomarkers have a critical role is new drug development. Drug development has consider- 
ably changed these last 20 years because impressive progresses in molecular biology have been made, leading to an increasingly detailed understanding of the genetic abnormalities in diseases [3]. The discovery of gene mutations and the identification of the cellular pathways that their encoded proteins control provide a wide range of new targets for drug development. Contemporary drug development is an ordered process starting with identification and validation of a target with high throughput methodologies and going through lead identification and optimization to preclinical and clinical trials of drug candidates [4]. In preclinical trials, the efficacy and toxicity of the drug is assessed in animals. Afterwards, the drug is tested in patients during three clinical phases. In phase 1, the toxicity, pharmacokinetics, pharmacodynamics, and optimal dose levels are assessed; in phase 2, the biological efficacy is determined; and in phase 3 , controlled trials are performed to assess the effects on clinical endpoints [5]. The term "pharmacokinetics" denotes what the body does to the drug in terms of its absorption, distribution, metabolism, and excretion, including the concentrationtime relationship and its dependence on dose, the term "pharmacodynamics" denotes what the drug does to the body [6].

Despite the successes with targeted drugs, there is a general concern about the slow arrival and increasing cost for development of new therapies. This is explained by the fact that the most reliable way to assess the clinical impact of a therapeutic intervention is through its effect on a clinical endpoint such as survival or disease-free survival. These standards may be impractical because long periods are required for these clinical endpoints to be achieved and trials with large number of patients are needed for their evaluation. The developments in quantitative medical imaging offer the opportunity of using imaging biomarkers to speed up the drug development process. For example, the lack of validated non-invasive biomarkers has been recognized as a major hinder to the development and use of antifibrotic drugs in patients with liver fibrosis [7].

Two types of biomarkers should be distinguished here:

- Pharmacodynamic biomarkers are used to show that the drug has an anatomical, functional, or biological effect on the target. Pharmacodynamic biomarkers are used in preclinical studies and phase 1 trials to confirm that the drug hits the target and that hitting this target alters the pathophysiological mechanism. The use of pharmacodynamic biomarkers in these early studies has the potential to improve patient selection, accelerate the drug development process, maximize the ability to generate important information about human disease, and decrease the risk of late and costly drug attrition $[8,9]$.
- Surrogate endpoints are biomarkers that are intended to substitute for a clinical endpoint and support approval for marketing authorization. They should thus be used in phase 2 and 3 trials to show that altering the pathophysiological mechanism of the disease affects the clinical status [10]. To substitute to a clinical endpoint such as progression free or overall survival, a surrogate endpoint has to be shown to be highly correlated with the clinical endpoint in a series of coordinated prospectively designed multi-centre trials culminating in a formal meta-analysis [11].

\section{Requirements for effective use of imaging biomarkers}

In addition to technology stability (robustness), noninvasiveness, and broad availability, there are two main prerequisites for the effective use of imaging biomarkers, which are standardization and validation.

\section{Standardization}

Standardization concerns the acquisition parameters for imaging and the post-processing methods. It is an essential step to ensure reproducibility across different centres and machines. Several consensus conferences have been held on standardization of perfusion MRI $[12,13]$ and diffusion MRI [14]. As the imaging and quantification methods are evolving, further update conferences are needed.

\section{Validation}

The sensitivity, specificity, precision, and reproducibility of new biomarkers have to be rigorously tested [15]. The tests should be conducted in phantoms, animals and humans [16]. The changes in biomarker values should be correlated to the biological effect and clinical endpoints (qualification). The validation requirements are much higher for surrogate endpoints than for pharmacodynamic biomarkers. Qualification as surrogate endpoints requires significant resources and will, in many cases, only be possible in a concerted effort of academia, industry and regulatory authorities, as proposed for example by the Biomarkers Consortium (http:// www.biomarkersconsortium.org), a public-private partnership managed by the Foundation for the National Institutes of Health in the USA.

\section{Types of biomarkers}

Anatomical, functional and molecular characteristics can be used as imaging biomarkers. In cancer treatment, CT (or 
MRI) measurements of changes of tumor volume are based on the "Response evaluation Criteria in Solid Tumours" (RECIST) criteria [17]. These anatomical biomarkers are the only imaging surrogate endpoints that are accepted in cancer treatment. However, these anatomical biomarkers are suboptimal to assess the effect of some targeted treatments that do not cause regression of tumor volume, but rather increase in the extent of tumor necrosis [18].

A lot of functional biomarkers obtained with several imaging methods, including PET, contrast-enhanced CT, perfusion CT and MRI, diffusion-weighted MRI, MR elastography and spectroscopy, have the potential to complement or even replace the RECIST criteria. However, important efforts of qualification and standardization remain to be done before the acceptance of some of these functional biomarkers as surrogate endpoints. ${ }^{18} \mathrm{~F}-\mathrm{FDG}$ PET may serve as an illustrative example. In clinical routine, ${ }^{18} \mathrm{~F}$-FDG-PET is increasingly used to assess the response to treatment in tumor patients. However, ${ }^{18} \mathrm{~F}$ FDG-PET as an imaging biomarker has not yet been sufficiently validated to be accepted as a surrogate endpoint for clinical studies by regulatory agencies. Currently, a multi-centre qualification study of ${ }^{18} \mathrm{~F}-\mathrm{FDG}-\mathrm{PET} / \mathrm{CT}$ as a predictive marker of tumor response and patient outcome in patients with non-small cell lung cancer has been initiated by the American College of Radiology Imaging Network (ACRIN).

Functional imaging biomarkers are increasingly used as pharmacodynamic biomarkers. Relative to molecular imaging biomarkers, which are target-specific, functional imaging biomarkers have the advantage of being downstream markers that probe general capabilities of disease, including cell death, proliferation, glycolysis, hypoxia, tumor invasiveness, angiogenesis, inflammation and fibrosis. Although a lot of functional imaging biomarkers have been proposed as pharmacodynamic biomarkers in early drug development, most of these biomarkers need further validation and standardization for their qualification in phase 1 and 2 human studies.

\section{Proposed actions}

The working group on imaging biomarkers recommends the creation of a European Network on Imaging Biomarkers (ENIB) under the umbrella of the European Institute for Biomedical Imaging research (EIBIR) and the European Society of Radiology (ESR) to coordinate, conduct and stimulate research and education about the development, standardization, validation, and qualification of imaging biomarkers at the European level. Extensive and comprehensive research in this field is often beyond the means of individual research groups and can best be accomplished through the multidisciplinary, multinational efforts of scientists. The cooperation of radiologists, nuclear medicine specialists, clinicians, and basic scientists in physics, biology, pathology, bioengineering, and mathematics is needed. The main tasks of ENIB will be:

- To coordinate the development of new biomarkers, especially biomarkers that probe hallmarks of diseases such as cell death, proliferation, glycolysis, hypoxia, tumor invasiveness, angiogenesis, inflammation and fibrosis.

- To provide standardization of the acquisition parameters for imaging and of the quantitative post-processing methods.

- To provide multi-centre validation and qualification of pharmacodynamic imaging biomarkers and surrogate endpoints.

- To stimulate comparative efficacy assessment between imaging biomarkers and other biomarkers, including histochemical and soluble biomarkers.

- To provide education about imaging biomarkers, including the creation of research fellowships programs in quantitative imaging and biomarkers.

The European Network on Imaging Biomarkers will work in cooperation with industry (pharmaceutical and medical device companies), European regulatory agencies (EMEA) [19], and international medical societies, including ECCO, EORTC, RSNA, EANM, ISMRM, ESMRMB and the subspeciality societies of the ESR for biomarker standardization, validation and qualification. The main purpose of ENIB will be to boost at the European level the development and use of qualified imaging biomarkers that will help in diagnosis, treatment assessment and drug development.

Acknowledgement This paper was prepared by the ESR working group on imaging biomarkers (Chairman: Van Beers B, Members: Cuenod CA, Marti-Bonmati L, Matos C, Niessen W, Padhani A). It was approved by the ESR Executive Council in March 2010.

\section{References}

1. Biomarkers definitions working group (2001) Biomarkers and surrogate endpoints: preferred definitions and conceptual framework. Clin Pharmacol Ther 69:89-95

2. Altar CA (2008) The biomarkers consortium: on the critical path of drug discovery. Clin Pharmacol Ther 83:361-364

3. Hanahan D, Weinberg R (2000) The hallmarks of cancer. Cell 100:57-70

4. Newell D (2003) The drug development process: from target discovery to the clinic. Clin Med 3:323-326

5. Pepe M, Etzioni R, Feng Z, Potter J, Lou M (2001) Phases of biomarkers development for early detection of cancer. J Natl Cancer Inst 93:1054-1061

6. Workman P, Aboagye E, Chung YL et al (2006) Minimally invasive pharmacokinetic and pharmacodynamic technologies in 
hypothesis-testing clinical trials of innovative therapies. J Natl Cancer Inst 98:580-598

7. Wells G (2009) Liver fibrosis: challenges of the new era. Gastroenterology 136:387-388

8. Kelloff G, Sigman C (2005) New science-based endpoints to accelerate oncology drug development. Eur J Cancer 41:491501

9. Carden CP, Banerji U, Kaye SB, Workman P, De Bono JS (2009) From darkness to light with biomarkers in early clinical trials of cancer drugs. Clin Pharmacol Ther 85:131-133

10. Ritcher W (2006) Imaging biomarkers as surrogate endpoints for drug development. Eur J Nucl Med Mol Imaging 33:S6-S10

11. Sargent DJ, Rubinstein L, Schwartz L et al (2009) Validation of novel imaging methodologies for use as cancer clinical trial endpoints. Eur J Cancer 45:290-299

12. Tofts PS, Brix G, Buckley DL et al (1999) Estimating kinetic parameters from dynamic contrast enhanced T1-weighted MRI of a diffusable tracer: standardized quantities and symbols. J Magn Reson Imaging 10:223-232

13. Leach MO, Brindle KM, Evelhoch JL et al (2005) The assessment of antiangiogenic and antivascular therapies in early-stage clinical trials using magnetic resonance imaging: issues and recommendations. Brit J Cancer 92:1599-1610

14. Padhani AR, Liu G, Mu-Koh D et al (2009) Diffusion weighted magnetic resonance imaging as a cancer biomarker: consensus and recommendations. Neoplasia 11:102-125

15. Van Beers BE, Vilgrain V (2009) Biomarkers in abdominal imaging. Abdom Imaging 34:663-667

16. Buckler AJ, Mozley PD, Schwartz L et al (2010) Volumetric CT in lung cancer: an example for the qualification of imaging as a biomarker. Acad Radiol 17:107-115

17. Eisenhauser EA, Therasse P, Bogaerts J et al (2009) New response evaluation criteria in solid tumours: revised RECIST guideline (version 1). Eur J Cancer 45:228-247

18. Llovet JM, Di Bisceglie A, Bruix J et al (2008) Design and endpoints of clinical trials in hepatocellular carcinoma. J Natl Cancer Inst 100:698-711

19. European Medicines Agency (2007) Innovative drug development approaches. Final report from the EMEA/CHMP-Think-Tank group on innovative drug development. Doc.Ref.: EMEA/ 127318/2007 available at http://www.emea.europa.eu/pdfs/human/ itf/12731807en.pdf 